\title{
Niveles de detección del plagio académico por el profesorado de educación secundaria: Análisis en función de sus áreas de conocimiento y medidas adoptadas*
}

Detection of levels of academic plagiarism by secondary school teachers: Analysis in relation to their fields of knowledge and measures adopted

Níveis de detecção de plágio acadêmico por professores do ensino secundário: Uma análise com base em suas áreas de conhecimento e as medidas tomadas

\author{
Mut-Amengual, Bartomeua ${ }^{a}$; Morey-López, Mercè ${ }^{b}$;ázquez-Recio, Rosa ${ }^{c}$
}

${ }^{a}$ Universitat Illes Balears, Islas Baleares, España. Teléfono: 0034-971172378.

Correo electrónico: tomeu.mut@uib.es

bUniversitat Illes Balears, Islas Baleares, España. Teléfono: 0034-9711172378.

Correo electrónico: merce.morey@uib.es

cUniversidad de Cádiz, Cádiz, España. Teléfono: 0034-956016732.

Correo electrónico: rmaria.vazquez@uca.es

\begin{abstract}
RESUMEN
Este trabajo analiza y describe, desde el punto de vista del profesorado, el fenómeno del plagio académico entre el alumnado de educación secundaria obligatoria de Andalucía y Baleares. Primero, se describen las frecuencias generales de detección de plagio señaladas por los docentes. Con posterioridad, se presentan los datos relativos a la asociación entre la frecuencia y prevalencia de detección del fenómeno por rama de conocimiento del profesorado y, en un tercer bloque, se detallan las medidas adoptadas por los docentes ante la sospecha de plagio. El estudio se ha llevado a cabo mediante la administración de un cuestionario a 453 docentes de secundaria de ambas comunidades autónomas. Los resultados obtenidos arrojan cifras relevantes, entre las que destacan las diferencias en los niveles de detección por parte de los docentes de distintas ramas de conocimiento. Esto permite la propuesta de acciones e iniciativas de intervención concretas, encaminadas a combatir dicho fenómeno.
\end{abstract}

Palabras clave: integridad académica, plagio académico, educación secundaria.

\begin{abstract}
This work analyses and describes, from the teachers' point of view, the phenomenon of academic plagiarism amongst compulsory secondary education students in Andalucía and Baleares. Firstly, the general frequency with which plagiarism is detected by teachers is described. Afterwards, data is presented relating to the association between the frequency and prevalence with which the phenomenon is detected according to the teachers' branch of knowledge. A third block details the measures taken by teachers suspecting plagiarism. The research used a questionnaire given to 453 secondary teachers in both autonomous communities. The results threw up some significant figures, amongst which the differences in detection levels by teachers in different subjects stand out. This makes a proposal for specific actions and initiatives directed at combating the phenomenon.
\end{abstract}

Key words: academic integrity, academic plagiarism, secondary education.

* Este trabajo se sitúa entre las actividades del proyecto de I+D titulado: El plagio académico entre el alumnado de Educación Secundaria Obligatoria de las Islas Baleares (Referencia EDU2009-14019-C02-01), financiado por la Dirección General de Investigación del Ministerio de Ciencia e Innovación del Gobierno de España. Los autores de este artículo forman parte del grupo de Investigación Educación y Ciudadanía de la Universidad de las Islas Baleares, que cuenta con la consideración de Grupo de Investigación Competitivo bajo el patrocinio de la Dirección General de Investigación, Desarrollo Tecnológico e Innovación de la Consejería de Innovación, Interior y Justicia del gobierno de la CAIB, y la cofinanciación de los Fondos FEDER. 


\section{RESUMO}

Analisa-se e descreve-se, do ponto de vista dos professores, o fenômeno do plágio acadêmico entre os estudantes da educação secundária obrigatória de Andalucía e Baleares. Primeiramente, descrevem-se as frequências gerais de detecção de plágio identificadas pelos professores. Posteriormente, apresentam-se os dados sobre a associação entre a frequência e a prevalência de detecção do fenômeno por áreas do conhecimento do professorado e, em um terceiro bloco, detalham-se as medidas tomadas pelos professores mediante a suspeita de plágio. O estudo foi realizado por meio de um questionário aplicado a 453 professores do Ensino Secundário das citadas comunidades autônomas. Resultados obtidos revelam números relevantes, entre os quais se destacam as diferenças entre os níveis de detecção por parte dos professores das diferentes áreas do conhecimento. Isso permite a proposta de ações e iniciativas de intervenções concretas para combater tal fenômeno.

Palavras chave: integridade acadêmica, plágio acadêmico, educação secundária.

\section{INTRODUCCIÓN}

El presente trabajo se adscribe al conjunto de investigaciones desarrolladas acerca de la integridad académica (Comas y Sureda, 2007; Comas, 2009; Mut, 2012). Señalar que bajo este concepto se engloban diferentes acciones o prácticas deshonestas desarrolladas por diferentes colectivos de la comunidad educativa, entre los que se encuentran el alumnado, el sector de administración y servicios académicos y el personal docente e investigador (Comas, 2009). Centrándonos en la praxis deshonesta cometida por el alumnado, y siguiendo a Sureda, Comas y Gili (2009), podemos poner de relieve varios ejemplos de este tipo de acciones: en el transcurso de un examen (copiar de un compañero/a, dejarse copiar, usar material no permitido; hacerse con las preguntas de la prueba antes de su realización, etc.), prácticas académicamente deshonestas en la elaboración y entrega de trabajos académicos (plagiar parcial o totalmente un trabajo, falsear datos en trabajos, etc.) o prácticas deshonestas en la vida académica diaria (dañar el mobiliario de la institución, faltar al respeto al resto de compañeros y personal de la institución, etc.). De todas las prácticas contra la integridad académica, la que más protagonismo ha alcanzado durante los últimos años es la que se ha venido a denominar "plagio académico" (Comas, 2009); es importante subrayar que durante las últimas décadas los trabajos en los que se analiza en profundidad este fenómeno han ido creciendo debido a la gran expectación e interés que ha generado este fenómeno en el mundo académico. Así, entre los trabajos pioneros en esta materia pueden destacarse las aportaciones realizadas por McCabe \& Trevino (1993), Hexham (1999), Jordan (2001) y Lambert, Hogan \& Barton (2003).

De entre todas las causas que se atribuyen al crecimiento de este fenómeno, en los últimos años sobresale en especial una de ellas: la irrupción de las tecnologías de la información y la comunicación (TIC) y, sobre todo, el uso generalizado de Internet como fuente de información entre el alumnado (Comas \& Sureda, 2010). Además, la facilidad y la comodidad que estas herramientas aportan en la edición y elaboración de textos (Sureda, Comas y Morey, 2009) han sido factores ciertamente desencadenantes del aumento en la comisión de estas acciones por parte del alumnado (Comas \& Sureda, 2010). Internet, de esta manera, se ha convertido en la principal fuente empleada a la hora de plagiar trabajos académicos por parte del alumnado universitario (Comas, Sureda, Casero y Morey, 2011), como también sucede en otras etapas o niveles educativos, como Secundaria o Bachillerato (Comas, Sureda, Angulo \& Mut, 2011; Mut, 2012; Morey, Sureda, Oliver y Comas, 2013).

La mayor parte de la literatura reciente en este campo se centra en analizar la extensión, las causas, las consecuencias y las maneras de prevenir el fenómeno en la etapa universitaria; 
en esta línea destacan los trabajos de McCabe, Trevino \& Butterfield (2001), Park (2003), Ercegovac \& Richardson (2004), Pupovac, Billic-Zulle \& Petrovecki (2008) y Comas, Sureda y Salvà (2010). Asimismo, si nos adentramos en niveles inferiores del sistema educativo observamos cómo el interés por este fenómeno disminuye considerablemente (Comas, Sureda, Angulo \& Mut, 2011). Aun así, encontramos trabajos que aportan interesantes datos acerca del plagio académico en etapas preuniversitarias -fundamentalmente Secundaria y Bachillerato-; estos aportes arrojan detalles clarificadores acerca de la prevalencia de este fenómeno en estas etapas. A modo de ejemplo, Branch (2001) señala que tres cuartas partes del alumnado preuniversitario admite plagiar, en al menos una ocasión, de forma regular. Villano (2006), Robelen (2007) y Ma, Wan \& Lu (2008), McCabe \& Katz (2009) señalan también unos porcentajes similares en la prevalencia de este fenómeno en dichas etapas. Estas cifras nos dan a entender que esta situación no se genera de manera esporádica en las aulas de educación superior, por lo que podemos entrever que la problemática arranca en ciclos previos a esta.

Gran parte de la literatura existente respectro del plagio académico se centra en analizar y describir la prevalencia de este fenómeno mediante aproximaciones realizadas sobre aportaciones del alumnado al respecto de este fenómeno y su comportamiento en relación con este (Comas, 2009). Por el contrario, existen escasos trabajos centrados, como el presente artículo, en analizar y describir este fenómeno desde la visión del profesorado. En nuestro caso, y partiendo de un estudio realizado conjuntamente entre la Universidad de Cádiz y la Universidad de las islas Baleares, este trabajo se basa en los datos obtenidos entre docentes de las islas Baleares y Andalucía, concretamente en la etapa de Secundaria, y los principales objetivos que nos proponemos se centran en: 1) determinar qué tipo de prácticas académicas deshonestas son las más prevalentes entre el alumnado de secundaria por medio de la experiencia del profesorado; 2) esclarecer si existe o no relación alguna entre el área de conocimiento a la que pertenecen los docentes y el haber detectado la comisión plagio entre su alumnado, y, 3) describir y analizar qué actuaciones llevan a cabo los docentes ante la sospecha de plagio académico (tanto desde un punto de vista general como partiendo del área de conocimiento de los mismos). En resumen, las preguntas de investigación (PI) a las que se pretende dar respuesta con este trabajo son:

- PI1: ¿Cuál es la frecuencia de comisión de plagio académico entre el alumnado de ESO según el profesorado en las CC.AA. de las islas Baleares y Andalucía y qué tipo de prácticas son las más frecuentes?

- PI2: ¿Existe relación entre la frecuencia en la detección de plagio académico y el área de conocimiento de las asignaturas que imparten los docentes encuestados?

- PI3: ¿Cuáles son las medidas adoptadas por el profesorado (a nivel general y en función del área de conocimiento) ante la sospecha o detección de casos de plagio académico entre su alumnado?

\section{MÉTODO}

\subsection{POBLACIÓN Y MUESTRA}

De las islas Baleares y de Andalucía 453 docentes de ESO han participado en el estudio, lo que supone una muestra con un margen de error de $\pm 2,6 \%$ para un intervalo de confianza 
estimado del $95 \%$ bajo la condición más desfavorable de $\mathrm{p}=\mathrm{q}=0,50^{1}$. El proceso de muestreo llevado a cabo fue de carácter aleatorio estratificado, teniendo en cuenta para ello: a) la impartición de docencia en los cuatro cursos de ESO (primero, segundo, tercero y cuarto); b) las provincias en el caso de Andalucía (Almería, Cádiz, Córdoba, Granada, Huelva, Jaén, Málaga y Sevilla) y la isla (Mallorca, Menorca e Ibiza-Formentera) en el caso de Baleares, y c) la titularidad de los centros (públicos y privados/concertados).

El cuestionario fue administrado de forma individual y anónima entre el profesorado de diferentes centros de ambas comunidades autónomas. Para ello se proporcionaron los cuestionarios a cada centro educativo para su posterior recogida una vez rellenados por los docentes. Los centros participantes fueron en las islas Baleares 37, y en Andalucía 19 (siendo del total 40 de titularidad pública y 16 concertados y privados). Es factible mencionar que el trabajo de campo (la distribución y posterior recogida de los cuestionarios) fue realizado por dos encuestadores previamente formados para llevar a cabo su tarea de forma eficiente (y consistente en informar acerca de los contenidos de la encuesta a los centros y dejar constancia de las incidencias en el momento de la recuperación del instrumento cumplimentado). La recogida de datos se llevó a cabo durante noviembrediciembre de 2011 y enero-febrero de 2012.

En cuanto a las características de los docentes participantes en el estudio, señalar que $43,8 \%$ pertenece al género masculino, mientras que $56,2 \%$ al género femenino. En cuanto a la edad, la mínima es de 25 años y la máxima de 65 , con una media de 41,97 años (con una desviación típica de 9,38).

\subsection{FUENTE DE DATOS Y VARIABLES}

El trabajo de campo fue realizado a partir de un cuestionario autoadministrado entre la muestra de docentes. Una vez diseñado el cuestionario se realizó una doble verificación: en primer lugar, este fue evaluado por siete profesionales (docentes de secundaria) y cinco investigadores expertos en métodos de investigación en ciencias sociales, indicando posibles modificaciones y valoraciones en diferentes ítems de la herramienta. Además se realizó una prueba piloto entre 12 docentes de ESO de las islas Baleares para comprobar la validez y la comprensión de este, y así de este modo asegurar la máxima adecuación de la herramienta a la muestra destinataria. Una vez recibidas las oportunas correcciones, valoraciones y estimaciones en las dos fases de comprobación se procedió a elaborar la herramienta definitiva: Cuestionario sobre plagio académico entre el alumnado de ESO. Así pues, finalmente, el cuestionario final utilizado en el trabajo de campo constaba de 17 preguntas (donde se incluían ítems de respuesta dicotómica, politómica e ítems de respuesta escala Likert) agrupadas en función de las distintas dimensiones analizadas en el estudio.

En particular, y partiendo de las tres preguntas de investigación (PI) de este trabajo, se han utilizado tres conjuntos de ítems que pasaremos a detallar a continuación. Estos son:

A. Pregunta de Investigación 1 (PI1). Se han analizado las respuestas obtenidas de los docentes en relación con las siguientes seis acciones planteadas:

1 Cálculo basado en los datos estadísticos del curso 2011-12 del Ministerio de Educación, Cultura y Deporte (2012). 
- Acción 1. Descubrir que un trabajo entregado por un/a alumno/a se trataba de una copia total de otro/a alumno/a realizado en cursos anteriores.

- Acción 2. Detectar que un trabajo entregado por un/a alumno/a contiene gran cantidad de fragmentos copiados de páginas web sin citar la fuente.

- Acción 3. Detectar que un trabajo entregado por un/a alumno/a contiene gran cantidad de fragmentos copiados de recursos impresos (libros, enciclopedias, diarios, artículos de revista, etc.) sin citar la fuente.

- Acción 4. Descubrir un trabajo copiado por completo de Internet y entregado, sin modificaciones, como un trabajo de asignatura.

- Acción 5. Descubrir un trabajo entregado por un/a alumno/a que contiene partes copiadas de páginas web que han sido traducidas al castellano o catalán y se ha presentado como elaboración propia.

- Acción 6. Descubrir un trabajo elaborado íntegramente a partir de fragmentos copiados literalmente de páginas web (sin que ninguna parte del trabajo haya sido escrita por el/la alumno/a).

Cada acción tenía una escala de respuesta que variaba entre: Nunca, Entre 1 y 2 veces, Entre 3 y 5 veces, Entre 6 y 10 veces y, por último, Más de 10 veces. Dicha escala ha permitido extraer los niveles de detección de prácticas fraudulentas entre el alumnado por parte del profesorado. Dichos niveles se exponen en este trabajo mediante los porcentajes (frecuencia general) en relación con las prácticas deshonestas percibidas por los docentes de Secundaria en función de cada una de las acciones contempladas en el cuestionario.

B. Pregunta de Investigación 2 (PI2). Se han relacionado las respuestas obtenidas de los docentes en relación a las 6 acciones planteadas en función del área de conocimiento de dichos docentes: Humanidades, Ciencias Sociales, Ciencias y Otras (Educación Física, Educación Musical, Tecnología, Religión, etc.).

C. Pregunta de Investigación 3 (PI3). Se han analizado las frecuencias (tanto a nivel general como en función del área de conocimiento del profesorado) de las medidas adoptadas por los docentes ante la sospecha de comisión de plagio académico entre el propio alumnado, siendo las opciones de respuesta las siguientes (de entre estas el profesorado podía escoger un máximo de 2):

- No hago nada

- Me pongo en contacto con el/la alumno/a y le comento mis sospechas, y que exprese su opinión

- Busco en Internet posibles fuentes de donde se ha podido plagiar el trabajo, por ejemplo, a partir de copiar y pegar en un buscador fragmentos sospechosos del trabajo

- Busco en libros, diccionarios, revistas, diarios y otros recursos impresos, posibles fuentes de donde se ha podido plagiar el trabajo

- Reviso referencias de las fuentes citadas en el trabajo

- Otra opción

\subsection{ANÁLISIS DE LOS DATOS}

La explotación de resultados se ha llevado a cabo mediante el paquete estadístico SPSS 21. En primer lugar, para el análisis de la primera cuestión planteada en el presente 
artículo (PI1) se ha realizado una explotación descriptiva en función de las frecuencias generales atribuidas a las seis acciones planteadas a los docentes, obteniendo de esta manera datos relativos a prevalencia de estas según el profesorado encuestado acerca del comportamiento del alumnado.

En referencia a la segunda cuestión analizada en este trabajo (P.I.2) se exponen y analizan los resultados en relación con las mismas seis acciones planteadas anteriormente, pero en este caso, correlacionadas con la rama de conocimiento a la que se adscriben los docentes. Para ello se ha llevado a cabo una explotación estadística mediante la prueba de correlación de Ji-Cuadrado de Pearson, la que permite la detección de relaciones estadísticamente significativas entre ambas variables.

Por último, para esclarecer y dar respuesta a la última cuestión planteada (P.I.3) respecto de las medidas adoptadas por el profesorado en caso de sospecha de trabajos plagiados, mencionar que se ha llevado a cabo un sumatorio de las frecuencias de cada una de las acciones señaladas entre los docentes acerca de su actuación en casos de esta índole, ya que se podían señalar dos opciones de respuesta. Posteriormente se ha definido el porcentaje correspondiente a cada una de ellas para determinar las acciones o medidas más señaladas entre los encuestados. A su vez, también se ha realizado un sumatorio de dichas respuestas en función del área de conocimiento del profesorado para evidenciar los niveles de detección en función de esta.

\section{RESULTADOS}

\subsection{PREVALENCIA DECLARADA POR EL PROFESORADO EN LA DETECCIÓN DE PRÁCTICAS ACADÉMICAS DESHONESTAS (FRECUENCIAS GENERALES)}

De los resultados obtenidos destaca la gran prevalencia de la Acción 2, 94,3\% del profesorado encuestado afirma haber detectado, al menos en una ocasión, prácticas de estas características durante el curso anterior a la administración del cuestionario. La segunda práctica más detectada, en al menos en una ocasión, es la Acción 6, con 76,1\%. Seguidamente se encuentran las acciones $4(70,9 \%), 5(62,1 \%), 3(58,9 \%)$, y finalmente, la Acción 1 (45,1\%).

También es factible destacar que hay docentes que coinciden en señalar que la acción detectada con más frecuencia (más de 10 veces durante el anterior curso académico) entre las seis acciones planteadas corresponde a la Acción 2: Detectar que un trabajo entregado por un alumno/a contiene gran cantidad de fragmentos copiados de páginas web sin citar la fuente; esta acción la señala $29 \%$ de los docentes para la frecuencia Más de 10 veces (véase Tabla 1). En segundo lugar observamos que la segunda acción más destacada corresponde a la Acción 6: Descubrir un trabajo elaborado íntegramente a partir de fragmentos copiados literalmente de páginas web (sin que ninguna parte del trabajo haya sido escrita por el alumno/a), con 12,1\% de docentes que afirman haber detectado este tipo de plagio en más de 10 ocasiones durante el curso 2010-2011. En tercer lugar se sitúa la Acción 5, con 9,4\%. Por último, las acciones menos frecuentes corresponden a las prácticas 4,3 y 1 . 
Tabla 1. Frecuencias obtenidas para cada una de las seis acciones analizadas en relación con la comisión de plagio académico

\begin{tabular}{|l|c|c|c|c|c|}
\hline \multicolumn{1}{|c|}{ Acción } & Nunca & $\begin{array}{c}\text { Entre } \\
\mathbf{1} \text { y 2 } \\
\text { veces }\end{array}$ & $\begin{array}{c}\text { Entre } \\
\mathbf{3} \text { y 5 } \\
\text { veces }\end{array}$ & $\begin{array}{c}\text { Entre } \\
\mathbf{6} \text { y 10 } \\
\text { veces }\end{array}$ & $\begin{array}{c}\text { Más } \\
\text { de 10 } \\
\text { veces }\end{array}$ \\
\hline $\begin{array}{l}\text { Acción 1. Descubrir que un trabajo entregado por un/a } \\
\text { alumno/a se trataba de una copia total de otro/a alumno/a } \\
\text { realizado en cursos anteriores }\end{array}$ & $54,9 \%$ & $30,5 \%$ & $9,7 \%$ & $3,6 \%$ & $1,4 \%$ \\
\hline $\begin{array}{l}\text { Acción 2. Detectar que un trabajo entregado por un/a } \\
\text { alumno/a contiene gran cantidad de fragmentos copiados } \\
\text { de páginas web sin citar la fuente }\end{array}$ & $5,7 \%$ & $19,3 \%$ & $27 \%$ & $19 \%$ & $29 \%$ \\
\hline $\begin{array}{l}\text { Acción 3. Detectar que un trabajo entregado por un/a } \\
\text { alumno/a contiene gran cantidad de fragmentos copia- } \\
\text { dos de recursos impresos (libros, enciclopedias, diarios, } \\
\text { artículos de revista, etc.) sin citar la fuente }\end{array}$ & $41,1 \%$ & $26,3 \%$ & $18,4 \%$ & $7,9 \%$ & $6,3 \%$ \\
\hline $\begin{array}{l}\text { Acción 4. Descubrir que un trabajo copiado por completo } \\
\text { de Internet y entregado, sin modificaciones, como un } \\
\text { trabajo de asignatura }\end{array}$ & $29,1 \%$ & $34,5 \%$ & $20 \%$ & $9,6 \%$ & $6,7 \%$ \\
\hline $\begin{array}{l}\text { Acción 5. Descubrir un trabajo entregado por un/a } \\
\text { alumno/a contiene partes copiadas de páginas web que } \\
\text { han sido traducidas al castellano o catalán y se ha pre- } \\
\text { sentado como elaboración propia }\end{array}$ & $37,9 \%$ & $27,6 \%$ & $16,1 \%$ & $9 \%$ & $9,4 \%$ \\
\hline $\begin{array}{l}\text { Acción 6. Descubrir un trabajo elaborado íntegramente } \\
\text { a partir de fragmentos copiados literalmente de páginas } \\
\text { web (sin que ninguna parte del trabajo haya sido escrita } \\
\text { por el/la alumno/a) }\end{array}$ & $23,9 \%$ & $28,8 \%$ & $22,1 \%$ & $13,2 \%$ & $12,1 \%$ \\
\hline
\end{tabular}

A su vez, atendiendo a las acciones que el profesorado señala haber detectado de forma más habitual (agrupando las respuestas que van Entre 6 y 10 veces y Más de 10 veces), se obtiene la siguiente clasificación ordenada de mayor a menor frecuencia de respuesta:

- Acción 2: Detectar que un trabajo entregado por un/a alumno/a contiene gran cantidad de fragmentos copiados de páginas web sin citar la fuente, con $48 \%$.

- Acción 6: Descubrir un trabajo elaborado íntegramente a partir de fragmentos copiados literalmente de páginas web (sin que ninguna parte del trabajo haya sido escrita por el/la alumno/a), con $25,3 \%$.

- Acción 5: Descubrir un trabajo entregado por un/a alumno/a contiene partes copiadas de páginas web que han sido traducidas al castellano o catalán y se ha presentado como elaboración propia, con $18,4 \%$.

- Acción 4: Descubrir un trabajo copiado por completo de Internet y entregado, sin modificaciones, como un trabajo de asignatura, con 16,3\%.

- Acción 3: Detectar que un trabajo entregado por un alumno/a contiene gran cantidad de fragmentos copiados de recursos impresos (libros, enciclopedias, diarios, artículos de revista, etc.) sin citar la fuente, con $14,2 \%$. 
- Acción 1: Descubrir que un trabajo entregado por un alumno/a se trataba de una copia total de otro/a alumno/a realizado en cursos anteriores, con 5\%.

\subsection{PREVALENCIA DECLARADA POR EL PROFESORADO EN LA DETECCIÓN DE PRÁCTICAS ACADÉMICAS DESHONESTAS (FRECUENCIA SEGÚN ÁREA DE CONOCIMIENTO)}

Siguiendo con la explotación de resultados y dando respuesta a la segunda cuestión abordada en este trabajo (PI2) se presentan los datos correspondientes a las acciones planteadas en función de la rama de conocimiento a la cual pertenece el profesorado (véanse Tablas 3 a 8).

Previamente, en la siguiente tabla (véase Tabla 2) se exponen los resultados derivados de la correlación de $\mathrm{Ji}$-Cuadrado de Pearson para el establecimiento de posibles relaciones entre cada acción detectada durante el curso 2010-2011 y la rama de conocimiento a la que pertenece el profesorado encuestado.

Tabla 2. Valores obtenidos mediante la prueba Ji-Cuadrado de Pearson

\begin{tabular}{|l|c|c|c|c|}
\hline \multicolumn{1}{|c|}{ Acción } & $\mathbf{n}$ & Valor & gl & $\mathbf{X}^{\mathbf{2}}$ \\
\hline $\begin{array}{l}\text { Acción 1. Descubrir que un trabajo entregado por un/a alumno/a se tra- } \\
\text { taba de una copia total de otro alumno/a realizado en cursos anteriores }\end{array}$ & 437 & 24,915 & 12 &, $015^{* 12}$ \\
\hline $\begin{array}{l}\text { Acción 2. Detectar que un trabajo entregado por un/a alumno/a } \\
\text { contiene gran cantidad de fragmentos copiados de páginas web sin } \\
\text { citar la fuente }\end{array}$ & 435 & 15,015 & 12 &, 241 \\
\hline $\begin{array}{l}\text { Acción 3. Detectar que un trabajo entregado por un/a alumno/a contie- } \\
\text { ne gran cantidad de fragmentos copiados de recursos impresos (libros, } \\
\text { enciclopedias, diarios, artículos de revista, etc.) sin citar la fuente }\end{array}$ & 439 & 25,023 & 12 &, $015^{* 3}$ \\
\hline $\begin{array}{l}\text { Acción 4. Descubrir un trabajo copiado por completo de Internet y } \\
\text { entregado, sin modificaciones, como un trabajo de asignatura }\end{array}$ & 440 & 20,624 & 12 &, 056 \\
\hline $\begin{array}{l}\text { Acción 5. Descubrir un trabajo entregado por un/a alumno/a contiene } \\
\text { partes copiadas de páginas web que han sido traducidas al castellano } \\
\text { o catalán y se ha presentado como elaboración propia }\end{array}$ & 440 & 15,236 & 12 &, 229 \\
\hline $\begin{array}{l}\text { Acción 6. Descubrir un trabajo elaborado íntegramente a partir de } \\
\text { fragmentos copiados literalmente de páginas web (sin que ninguna } \\
\text { parte del trabajo haya sido escrita por el/la alumno/a) }\end{array}$ & 442 & 15,722 & 12 &, 204 \\
\hline
\end{tabular}

$\mathrm{n}=$ número de profesores de la muestra; Valor (Ji-Cuadrado); gl= grados de libertad; $\mathrm{X}^{2}=$ significación bilateral, valor obtenido por cada grupo, *Significativa al nivel 0,05

Los datos ponen de manifiesto que existe relación directa entre la rama de conocimiento y la frecuencia de detección de algunas de las prácticas académicamente deshonestas planteadas, concretamente con las acciones 1 y 3 .

2 Existen diferencias estadísticamente significativas entre la acción analizada y la variable rama de conocimiento. 
En el caso de la Acción 1, y como puede apreciarse en la Tabla 3, las diferencias entre los niveles de identificación y la rama de conocimiento se focalizan especialmente en los porcentajes de respuesta dada por los docentes de Humanidades y Ciencias Sociales frente a los de Ciencias y Otros, especialmente en la opción "Nunca". Así pues, los dos primeros grupos de docentes presentan porcentajes de respuesta para la opción Nunca muy inferiores al resto: 48,1\% y 46,3\% frente al 61,7\% de Ciencias y al 64,3\% de otras áreas de conocimiento (Educación Física, Educación Musical, Tecnología, Religión, etc.). En definitiva, estos porcentajes evidencian que existe una mayor propensión del profesorado de Humanidades y Ciencias Sociales (frente al de Ciencias y otras áreas de conocimiento) a identificar trabajos entregados por un alumno que ya habían sido entregados anteriormente por otro.

Tabla 3. Frecuencias obtenidas para la Acción 1 en función de la rama de conocimiento

\begin{tabular}{|l|c|c|c|c|c|}
\hline $\begin{array}{l}\text { Acción 1. Descubrir que un trabajo entregado por } \\
\text { un/a alumno/a se trataba de una copia total de otro/a } \\
\text { alumno/a realizado en cursos anteriores }\end{array}$ & Nunca & $\begin{array}{c}\text { Entre } \\
\mathbf{1} \text { y } 2 \\
\text { veces }\end{array}$ & $\begin{array}{c}\text { Entre } \\
\mathbf{3} \text { y 5 } \\
\text { veces }\end{array}$ & $\begin{array}{c}\text { Entre } \\
\mathbf{6} \text { y 10 } \\
\text { veces }\end{array}$ & $\begin{array}{c}\text { Más } \\
\text { de 10 } \\
\text { veces }\end{array}$ \\
\hline Humanidades & $48,1 \%$ & $31,6 \%$ & $13,3 \%$ & $7 \%$ & $0 \%$ \\
\hline Ciencias Sociales & $46,3 \%$ & $41,8 \%$ & $10,4 \%$ & $0 \%$ & $1,5 \%$ \\
\hline Ciencias & $61,7 \%$ & $25,8 \%$ & $7,8 \%$ & $2,3 \%$ & $2,3 \%$ \\
\hline $\begin{array}{l}\text { Otros (Educación Física, Educación Musical, } \\
\text { Tecnología, Religión, etc.) }\end{array}$ & $64,3 \%$ & $25 \%$ & $6 \%$ & $2,4 \%$ & $2,4 \%$ \\
\hline
\end{tabular}

Paralelamente, y tal y como se desprende de la Tabla 5, el índice de significación localizado en la Acción 3 se corresponde con la baja identificación de trabajos con fragmentos copiados de recursos impresos por parte del profesorado de Ciencias (con un porcentaje del 51,2\% que afirma que Nunca los ha detectado) frente al profesorado de otras áreas de conocimiento que declara haber identificado este tipo de prácticas en al menos una ocasión en $73,5 \%$ de los casos.

De hecho, un análisis conjunto de ambas acciones (Acción 1 y Acción 3) evidencia, a nivel general, una menor propensión del profesorado de Ciencias a identificar acciones académicamente deshonestas relacionadas con el plagio de fuentes impresas, ya sea de trabajos anteriores de otros alumnos o bien de recursos académicos impresos (libros, enciclopedias, artículos de revista, etc.).

Así pues, no se ha establecido ningún tipo de relación estadísticamente significativa entre la rama de conocimiento y las acciones $2,4,5$ y 6 , lo que significa que el profesorado, indistintamente de la rama de conocimiento a la que pertenece, presenta frecuencias en la detección de este tipo de acciones de manera similar. Igualmente (véanse Tablas 4, 6, 7 y 8), además de evidenciarse un nivel de identificación homogéneo en función del área de conocimiento, los datos también muestran unas cotas de detección mucho más elevadas: mientras que en la Acción 1, por ejemplo, 61,7\% del profesorado de Ciencias decía que Nunca había detectado este tipo de prácticas, el máximo al que 
este porcentaje asciende en cualquiera de las acciones que no presentan diferencias estadísticamente significativas es $41,4 \%$ para la misma opción de respuesta (Acción 5).

Tabla 4. Frecuencias obtenidas para la Acción 2 en función de la rama de conocimiento

\begin{tabular}{|l|l|l|l|l|l|}
\hline $\begin{array}{l}\text { Acción 2. Detectar que un trabajo entregado por } \\
\text { un/a alumno/a contiene gran cantidad de fragmentos } \\
\text { copiados de páginas web sin citar la fuente }\end{array}$ & Nunca & $\begin{array}{c}\text { Entre } \\
\mathbf{1} \text { y 2 } \\
\text { veces }\end{array}$ & $\begin{array}{c}\text { Entre } \\
\mathbf{3} \text { y } \mathbf{5} \\
\text { veces }\end{array}$ & $\begin{array}{c}\text { Entre } \\
\mathbf{6} \text { y 10 } \\
\text { veces }\end{array}$ & $\begin{array}{c}\text { Más } \\
\text { de 10 } \\
\text { veces }\end{array}$ \\
\hline Humanidades & $4,4 \%$ & $21,9 \%$ & $31,2 \%$ & $20 \%$ & $22,5 \%$ \\
\hline Ciencias Sociales & $1,5 \%$ & $16,4 \%$ & $23,9 \%$ & $20,9 \%$ & $37,3 \%$ \\
\hline Ciencias & $6,2 \%$ & $19,5 \%$ & $23,4 \%$ & $14,8 \%$ & $35,9 \%$ \\
\hline $\begin{array}{l}\text { Otros (Educación Física, Educación Musical, Tecnología, } \\
\text { Religión, etc.) }\end{array}$ & $8,8 \%$ & $17,5 \%$ & $27,5 \%$ & $21,2 \%$ & $25 \%$ \\
\hline
\end{tabular}

Tabla 5. Frecuencias obtenidas para la Acción 3 en función de la rama de conocimiento

\begin{tabular}{|l|c|c|c|c|c|}
\hline $\begin{array}{l}\text { Acción 3. Detectar que un trabajo entregado por } \\
\text { un/a alumno/a contiene gran cantidad de fragmentos } \\
\text { copiados de recursos impresos (libros, enciclopedias, } \\
\text { diarios, artículos de revista, etc.) sin citar la fuente }\end{array}$ & Nunca & $\begin{array}{c}\text { Entre } \\
\mathbf{1} \text { y } \mathbf{2} \\
\text { veces }\end{array}$ & $\begin{array}{c}\text { Entre } \\
\mathbf{3} \text { y 5 } \\
\text { veces }\end{array}$ & $\begin{array}{c}\text { Entre } \\
\mathbf{6} \text { y 10 } \\
\text { veces }\end{array}$ & $\begin{array}{c}\text { Más } \\
\text { de 10 } \\
\text { veces }\end{array}$ \\
\hline Humanidades & $44,4 \%$ & $28,7 \%$ & $17,5 \%$ & $5 \%$ & $4,4 \%$ \\
\hline Ciencias Sociales & $31,3 \%$ & $22,4 \%$ & $26,9 \%$ & $10,4 \%$ & $9 \%$ \\
\hline Ciencias & $51,2 \%$ & $20,9 \%$ & $14,7 \%$ & $5,4 \%$ & $7,8 \%$ \\
\hline $\begin{array}{l}\text { Otros (Educación Física, Educación Musical, Tecnología, } \\
\text { Religión, etc.) }\end{array}$ & $26,5 \%$ & $34,9 \%$ & $20,5 \%$ & $12 \%$ & $6 \%$ \\
\hline
\end{tabular}

Tabla 6. Frecuencias obtenidas para la Acción 4 en función de la rama de conocimiento

\begin{tabular}{|l|c|c|c|c|c|}
\hline $\begin{array}{l}\text { Acción 4. Descubrir un trabajo copiado por completo } \\
\text { de Internet y entregado, sin modificaciones, como un } \\
\text { trabajo de asignatura }\end{array}$ & Nunca & $\begin{array}{c}\text { Entre } \\
\mathbf{1} \text { y } 2 \\
\text { veces }\end{array}$ & $\begin{array}{c}\text { Entre } \\
\mathbf{3} \text { y 5 } \\
\text { veces }\end{array}$ & $\begin{array}{c}\text { Entre } \\
\mathbf{6} \text { y 10 } \\
\text { veces }\end{array}$ & $\begin{array}{c}\text { Más } \\
\text { de 10 } \\
\text { veces }\end{array}$ \\
\hline Humanidades & $24,1 \%$ & $40,1 \%$ & $21 \%$ & $9,9 \%$ & $4,9 \%$ \\
\hline Ciencias Sociales & $19,4 \%$ & $31,3 \%$ & $23,9 \%$ & $16,4 \%$ & $9 \%$ \\
\hline Ciencias & $37,5 \%$ & $32,8 \%$ & $14,8 \%$ & $6,2 \%$ & $8,6 \%$ \\
\hline $\begin{array}{l}\text { Otros (Educación Física, Educación Musical, } \\
\text { Tecnología, Religión, etc.) }\end{array}$ & $33,7 \%$ & $28,9 \%$ & $24,1 \%$ & $9,6 \%$ & $3,6 \%$ \\
\hline
\end{tabular}


Tabla 7. Frecuencias obtenidas para la Acción 5 planteada en función de la rama de conocimiento

\begin{tabular}{|l|l|l|l|l|l|}
\hline $\begin{array}{l}\text { Acción 5. Descubrir que un trabajo entregado por } \\
\text { un/a alumno/a contiene partes copiadas de páginas } \\
\text { web que han sido traducidas al castellano o catalán } \\
\text { y se ha presentado como elaboración propia }\end{array}$ & Nunca & $\begin{array}{c}\text { Entre } \\
\mathbf{1} \text { y } 2 \\
\text { veces }\end{array}$ & $\begin{array}{c}\text { Entre } \\
\mathbf{3} \text { y 5 } \\
\text { veces }\end{array}$ & $\begin{array}{c}\text { Entre } \\
\mathbf{6} \text { y 10 } \\
\text { veces }\end{array}$ & $\begin{array}{c}\text { Más } \\
\text { de 10 } \\
\text { veces }\end{array}$ \\
\hline Humanidades & $37,7 \%$ & $28,4 \%$ & $17,3 \%$ & $9,9 \%$ & $6,8 \%$ \\
\hline Ciencias Sociales & $32,8 \%$ & $31,3 \%$ & $13,4 \%$ & $11,9 \%$ & $10,4 \%$ \\
\hline Ciencias & $41,4 \%$ & $28,1 \%$ & $11,7 \%$ & $4,7 \%$ & $14,1 \%$ \\
\hline $\begin{array}{l}\text { Otros (Educación Física, Educación Musical, Tecnología, } \\
\text { Religión, etc.) }\end{array}$ & $36,1 \%$ & $22,9 \%$ & $24,1 \%$ & $9,6 \%$ & $7,2 \%$ \\
\hline
\end{tabular}

Tabla 8. Frecuencias obtenidas para la Acción 6 planteada en función de la rama de conocimiento

\begin{tabular}{|l|c|c|c|c|c|}
\hline $\begin{array}{l}\text { Acción 6. Descubrir un trabajo elaborado íntegramente } \\
\text { a partir de fragmentos copiados literalmente de } \\
\text { páginas web (sin que ninguna parte del trabajo haya } \\
\text { sido escrita por el/la alumno/a) }\end{array}$ & Nunca & $\begin{array}{c}\text { Entre } \\
\mathbf{1} \text { y 2 } \\
\text { veces }\end{array}$ & $\begin{array}{c}\text { Entre } \\
\mathbf{3} \text { y 5 } \\
\text { veces }\end{array}$ & $\begin{array}{c}\text { Entre } \\
\mathbf{6} \text { y 10 } \\
\text { veces }\end{array}$ & $\begin{array}{c}\text { Más } \\
\text { de 10 } \\
\text { veces }\end{array}$ \\
\hline Humanidades & $22,6 \%$ & $28 \%$ & $28 \%$ & $12,8 \%$ & $8,5 \%$ \\
\hline Ciencias Sociales & $22,4 \%$ & $32,8 \%$ & $17,9 \%$ & $17,9 \%$ & $9 \%$ \\
\hline Ciencias & $26,6 \%$ & $25 \%$ & $18 \%$ & $11,7 \%$ & $18,8 \%$ \\
\hline $\begin{array}{l}\text { Otros (Educación Física, Educación Musical, Tecnología, } \\
\text { Religión, etc.) }\end{array}$ & $21,7 \%$ & $34,9 \%$ & $21,7 \%$ & $10,8 \%$ & $10,8 \%$ \\
\hline
\end{tabular}

Asimismo, si el análisis se centra en aquellas acciones que el profesorado señala haber detectado de forma más frecuente (entre 6 y 10 veces y más de 10 veces), obtenemos los resultados siguientes para cada acción por orden descendente en función de la frecuencia de respuesta:

- Para la Acción 1 destacar que el profesorado de Humanidades manifiesta haber identificado esta práctica en $7 \%$, seguido por el profesorado de otras disciplinas con $4,8 \%$, Ciencias $4,6 \%$ y Ciencias Sociales ocupa el último lugar con $1,5 \%$.

- En la Acción 2, 58,2\% del profesorado de Ciencias Sociales manifiesta haber detectado prácticas de esta índole, el profesorado de Ciencias en 50,7\%, otras disciplinas 46,2\% y, por último, hallamos al profesorado de Humanidades con 42,5\%.

- En la Acción 3, 19,4\% del profesorado de Ciencias Sociales señala haber detectado esta acción, $18 \%$ de otras disciplinas, 13,2\% corresponde al profesorado de Ciencias, y, en último lugar, encontramos el profesorado de Humanidades con 9,4\%.

- En la Acción 4, nuevamente en primer lugar encontramos el profesorado de Ciencias Sociales con $25,4 \%$ que afirma haber detectado esta práctica, seguido por del de Ciencias y Humanidades con un porcentaje para ambos del 14,8\% y, por último, el profesorado de otras disciplinas con $13,2 \%$. 
- En la Acción 5, en primera posición se encuentra de nuevo el profesorado perteneciente a la rama de conocimiento de Ciencias Sociales con $22,3 \%$, seguido por el de Ciencias con $18,8 \%$, continuado con el de otras disciplinas con $16,8 \%$ y, en último lugar, el profesorado perteneciente a la rama de Humanidades con 16,7\%.

- Finalmente, para la Acción 6 encontramos con mayor índice de prácticas observadas de este tipo al profesorado de Ciencias con 30,5\%, seguido por los docentes de Ciencias Sociales con $26,9 \%$, el profesorado de otras disciplinas con $21,6 \%$ y, para finalizar, el profesorado con menor detección en prácticas de estas características se sitúan los docentes de Humanidades con $21,3 \%$.

\subsection{NIVELES DE ACTUACIÓN DEL PROFESORADO EN CASO DE SOSPECHA DE PLAGIO ACADÉMICO (FRECUENCIAS GENERALES Y EN FUNCIÓN DEL ÁREA DE CONOCIMIENTO)}

A continuación se presentan los resultados obtenidos en relación con la tercera y última cuestión planteada en este trabajo (PI3) relativa, en primer lugar, a las medidas adoptadas por el profesorado ante casos sospechosos de plagio académico entre su alumnado (véase Tabla 9) y, a continuación, en función del área de conocimiento de los docentes (véase Tabla 10). Así, en el cuestionario se planteaban diferentes opciones de respuesta (todas ellas referidas a medidas de actuación ante la sospecha de que su alumnado hubiera cometido fraude académico), de estas los docentes podían señalar las dos más significativas.

Tabla 9. Frecuencias obtenidas de las medidas adoptadas por el profesorado (resultados generales ordenados por frecuencia)

\begin{tabular}{|l|c|c|}
\hline \multicolumn{1}{|c|}{ Medidas } & Frecuencia (N) & Porcentaje \\
\hline $\begin{array}{l}\text { Ponerse en contacto con el/la alumno/a para comentar las } \\
\text { sospechas }\end{array}$ & 375 & 48,2 \\
\hline Buscar en Internet posibles fuentes de plagio & 255 & 32,7 \\
\hline Revisar las referencias de las fuentes citadas en el trabajo & 84 & 10,8 \\
\hline Otras & 40 & 5,1 \\
\hline Buscar en libros, diccionarios, revistas, diarios y otros recursos & 18 & 2,3 \\
\hline Nada & 7 & 0,9 \\
\hline Total & 779 & 100 \\
\hline
\end{tabular}

Es destacable que casi la mitad de las respuestas dadas por el profesorado participante, $48,2 \%$, se refiere a ponerse en contacto con el alumno/a para intentar esclarecer las sospechas suscitadas por el trabajo presentado. Como segunda opción más señalada encontramos la referente a buscar en Internet posibles fuentes de plagio para dar respuesta a sus sospechas, con un porcentaje de respuesta del 32,7\%. En tercer lugar, los docentes encuestados señalan que acuden a las referencias citadas en los trabajos para corroborar o desmentir sus sospechas (10,8\% así lo manifiesta). Destacar que en las últimas posiciones 
se encuentra la acción referida a la utilización de soportes impresos tradicionales como libros, revistas, diccionarios, etc., con $2,3 \%$.

A su vez, la respuesta obtenida del profesorado ante la sospecha de casos de plagio académico en función del área de conocimiento a la que se adscribe evidencia algunas leves diferencias (véase Tabla 10).

Tabla 10. Frecuencias obtenidas de las medidas adoptadas por el profesorado (en función de la rama de conocimiento)

\begin{tabular}{|c|c|c|c|c|c|c|}
\hline & $\begin{array}{c}\text { Ponerse en } \\
\text { contacto } \\
\text { con el/la } \\
\text { alumno/a para } \\
\text { comentar las } \\
\text { sospechas } \\
\end{array}$ & $\begin{array}{c}\text { Buscar en } \\
\text { Internet } \\
\text { posibles } \\
\text { fuentes de } \\
\text { plagio }\end{array}$ & $\begin{array}{c}\text { Revisar las } \\
\text { referencias } \\
\text { de las fuentes } \\
\text { citadas en el } \\
\text { trabajo }\end{array}$ & $\begin{array}{c}\text { Buscar } \\
\text { en libros, } \\
\text { diccionarios, } \\
\text { revistas, } \\
\text { diarios y otros } \\
\text { recursos }\end{array}$ & Nada & Otras \\
\hline $\begin{array}{l}\text { Humanidades } \\
\mathrm{N}=292\end{array}$ & $\begin{array}{c}45,5 \% \\
(n=133)\end{array}$ & $\begin{array}{l}35,9 \% \\
(n=105)\end{array}$ & $\begin{array}{c}7,8 \% \\
(n=23)\end{array}$ & $\begin{array}{l}3,4 \% \\
(\mathrm{n}=10)\end{array}$ & $\begin{array}{l}1,02 \% \\
(\mathrm{n}=3)\end{array}$ & $\begin{array}{c}6,1 \% \\
(\mathrm{n}=18)\end{array}$ \\
\hline $\begin{array}{l}\text { Ciencias } \\
\text { Sociales } \\
\mathrm{N}=113\end{array}$ & $\begin{array}{l}54,8 \% \\
(\mathrm{n}=62)\end{array}$ & $\begin{array}{l}27,4 \% \\
(n=31)\end{array}$ & $\begin{array}{c}9,7 \% \\
(\mathrm{n}=11)\end{array}$ & $\begin{array}{l}1,7 \% \\
(n=2)\end{array}$ & $\begin{array}{c}0,88 \% \\
(\mathrm{n}=1)\end{array}$ & $\begin{array}{l}5,3 \% \\
(\mathrm{n}=6)\end{array}$ \\
\hline $\begin{array}{l}\text { Ciencias } \\
\mathrm{N}=224\end{array}$ & $\begin{array}{l}48,2 \% \\
(n=108)\end{array}$ & $\begin{array}{l}30,8 \% \\
(n=69)\end{array}$ & $\begin{array}{l}14,7 \% \\
(n=33)\end{array}$ & $\begin{array}{l}2,2 \% \\
(\mathrm{n}=5)\end{array}$ & $\begin{array}{l}0,44 \% \\
(\mathrm{n}=1)\end{array}$ & $\begin{array}{l}3,5 \% \\
(\mathrm{n}=8)\end{array}$ \\
\hline $\begin{array}{l}\text { Otros } \\
N=139\end{array}$ & $\begin{array}{l}47,4 \% \\
(n=66)\end{array}$ & $\begin{array}{l}33,1 \% \\
(\mathrm{n}=46)\end{array}$ & $\begin{array}{l}11,5 \% \\
(n=16)\end{array}$ & $\begin{array}{l}0,7 \% \\
(\mathrm{n}=1)\end{array}$ & $\begin{array}{l}1,4 \% \\
(\mathrm{n}=2)\end{array}$ & $\begin{array}{l}5,7 \% \\
(\mathrm{n}=8)\end{array}$ \\
\hline
\end{tabular}

Partiendo del análisis de los resultados a partir de las opciones de respuesta se constata que el profesorado que opta principalmente por contactar directamente con el alumnado para comunicarle sus sospechas es el adscrito al área de Ciencias Sociales $(54,8 \%)$, mientras que los docentes que menos han señalado esta respuesta han sido los de Humanidades (45,5\%). También esta es el área de conocimiento (Humanidades) que presenta mayores porcentajes de respuesta en la opción Nada (con $1,02 \%$, solo superado por el $1,4 \%$ del profesorado adscrito a "otras" ramas de conocimiento).

En lo que respecta a la revisión de las fuentes que se han utilizado en los trabajos en los que se sospecha que se ha cometido plagio académico, es el profesorado de Ciencias el que más señala esta opción (con 14,7\%), siendo el adscrito al área de Humanidades el que menos $(7,8 \%)$.

Finalmente, y relativo a la búsqueda de fuentes de plagio en documentos impresos o bien electrónicos, en ambos casos destaca el profesorado de Humanidades por ser el que más señala cada una de estas opciones, con 3,4\% y 35,9\% respectivamente, a la vez que es el profesorado adscrito a Ciencias Sociales el colectivo que menos acude a fuentes electrónicas para contrastar esta información $(27,4 \%)$. 


\section{LIMITACIONES, CONCLUSIONES Y DISCUSIÓN}

El presente trabajo, como ha quedado reflejado en el apartado metodológico, posee las características óptimas para permitir la realización de generalizaciones, porque a nivel muestral se parte de un tamaño y composición válidos y adecuados. Si bien es cierto que el hecho de ceñir el estudio a la frecuencia general y la correlación de resultados según la rama de conocimiento y las acciones punibles planteadas por parte del alumnado, según la visión del profesorado, puede comportar leves limitaciones al estudio.

Debe entenderse este trabajo como una aproximación a un fenómeno complejo, como es el del plagio académico, con múltiples elementos asociados e interrelacionados que lo configuran. De este modo, este trabajo pretende ayudar a entender y esclarecer este fenómeno para poder aportar soluciones válidas y que supongan una mejora en la práctica docente.

A partir del análisis de los resultados recogidos pueden deducirse algunas conclusiones y reflexiones que invitan al planteamiento de propuestas de actuación en la etapa educativa de Educación Secundaria para intentar atenuar este fenómeno. En primer lugar, ciñéndonos a los datos desprendidos en las frecuencias generales (PI1) acerca de los tipos de acciones detectadas por el profesorado, observamos la existencia de frecuencias dispares en las tipologías de comisión de plagio académico detectadas, aunque de estas es valioso destacar de manera clara la Acción número 2, que supone el porcentaje más elevado respecto de las demás acciones. Esta es la práctica entendida como plagio collage (Comas, 2009), consistente en la elaboración de trabajos a partir de fragmentos copiados de Internet sin citar la fuente. De hecho, y como se ha destacado anteriormente, corresponden a las Acciones 1 y 3 los niveles más bajos de detección de este tipo de prácticas (las prácticas relacionadas con la elaboración de trabajos a partir de fuentes impresas) por parte del profesorado de Andalucía y Baleares, esto resulta lógico si tenemos en cuenta que Internet es la principal fuente de documentación académica (Comas \& Sureda, 2010), a la vez que también es el recurso más utilizado para la realización de prácticas académicamente deshonestas debido a las facilidades de edición de texto que presenta el formato electrónico (DeBell \& Chapman, 2003; Ercegovac \& Richardson, 2004; Embleton \& Helfer, 2007; Comas \& Sureda, 2010). Además, igualmente esta también resulta ser la tipología de plagio más fácilmente detectable por los docentes gracias al uso de las tecnologías de la información y la comunicación, por lo que no es de extrañar que se den estos altos índices de prevalencia en su identificación por parte del profesorado. También es sustancial remarcar, además, que un elemento más de validación de los resultados obtenidos en cuanto a los altos niveles de detección de determinadas prácticas (aquellas relativas al ciberplagio) entre el profesorado se hallan en consonancia con los niveles revelados en trabajos previos centrados exclusivamente en el alumnado y que recogían los niveles de comisión de prácticas académicamente deshonestas por parte de este (Kisamore, Stone \& Jawahar, 2007; Davis, Drinan \& Bertram-Gallant, 2009; Ma, McCabe \& Liu, 2013).

La segunda cuestión que se aborda en este trabajo (PI2), donde se analiza la existencia de correlación estadística entre el nivel de detección del plagio académico y la rama de conocimiento a la que pertenece el profesorado, arroja datos ciertamente interesantes. Las Acciones 2, 4, 5 y 6 no desprenden significación estadística, hecho que nos muestra que no existe ningún tipo de diferencia en relación con el nivel de detección en la comisión de estas prácticas entre los alumnos en función de la rama de conocimiento a la 
que pertenece el profesorado. Este factor es claramente significativo, ya que estas cuatro acciones hacen referencia a prácticas o conductas relacionadas con el uso de Internet. Además, si se analizan los resultados extraídos para las Acciones 1 y 3 (aquellas que sí presentan significación estadística) en función del área de conocimiento de los docentes, son los de Humanidades los que presentan valores más bajos en la opción de respuesta Nunca para la Acción 1, mientras que el profesorado de Ciencias Sociales es el que muestra un valor más elevado en la detección de la Acción 3 de forma más frecuente (entre 6 y 10 veces y más de 10 veces). Ciertamente, ello nos induce a extraer que es la tipología de trabajos exigidos al alumnado un elemento que determina los índices de comisión (y por tanto de detección) de este tipo de prácticas académicamente deshonestas. Estas cifras, de hecho, obtienen mayor trascendencia si las comparamos con otros estudios que arrojan cifras similares. En esta dirección, el estudio de Morey et al. (2013) acerca de características análogas desprende cifras que van en consonancia con los resultados aportados en esta investigación.

En la tercera dimensión planteada en este estudio (PI3), respecto de las medidas para la detección tomadas por el profesorado de Andalucía y Baleares ante un posible caso de plagio académico (tanto a nivel general como por área de conocimiento), subrayar que casi la mitad de estos, como primera opción, optan por conversar cara a cara con el alumnado relativo a las sospechas generadas por dicho trabajo, siendo el profesorado de Ciencias Sociales el que más destaca por abordar de esta manera la problemática $(54,8 \%)$. Por lo demás, resulta conveniente señalar que del mismo modo que Internet ha servido para potenciar este fenómeno, también ha servido para facilitar la detección de estas prácticas mediante la utilización de motores de búsqueda; así pues, Internet se ha convertido en una herramienta básica para el profesorado a la hora de realizar comprobaciones frente a las sospechas generadas por trabajos de dudosa originalidad; casi un tercio de los docentes manifiesta utilizar el foco de información -Internet- para verificar o desmentir sus sospechas como segunda opción, destacando los del área de Humanidades por emplear más comúnmente esta herramienta $(35,9 \%)$. Estas dos acciones suponen más del $80 \%$ de las medidas tomadas. Del $20 \%$ restante, destacar un resultado que cuanto menos llama la atención: $0,9 \%$-es decir, un docente de cada cien- afirma no hacer nada ante un posible caso de plagio académico, siendo el profesorado de Ciencias el que más medidas toma (de un tipo o de otro) frente a la detección de algún caso (únicamente el $0,44 \%$ de los docentes de este grupo admite no hacer nada en absoluto ante las sospechas). Afortunadamente, este último dato no se asemeja a los obtenidos por estudios recientes en el ámbito universitario (Coren, 2011), en los que un 40\% de los docentes admite ignorar conductas académicamente deshonestas detectadas entre su alumnado, lo que consideramos, al igual que Volpe, Davidson \& Bell (2008), que únicamente puede tener efectos negativos, ya que esta falta de corrección o sanción influye directamente en los niveles de comisión. De hecho, como ya evidenciaban Zopatis \& Krambia-Kapardis (2008), si la cultura de la institución educativa apoya el comportamiento académicamente deshonesto (ya sea ignorando las evidencias o simplemente no contando con una normativa clara que contemple los casos de fraude), los estudiantes considerarán dicha deshonestidad como algo aceptable y apropiado.

Todos estos datos, como decíamos, deben resultarnos útiles para definir y plantear medidas eficaces y eficientes para combatir este fenómeno. Así pues, algunos planteamientos prácticos podrían ser: 
- Diseñar y proponer al alumnado trabajos académicos que no permitan la reproducción de conductas académicamente deshonestas (como la entrega de trabajos ya presentados anteriormente), y potenciar así la elaboración de trabajos originales basados en el análisis y resolución de problemas, como sucede en el caso de los que se plantean por parte de los docentes adscritos al área de Ciencias, por ejemplo.

- Proporcionar al alumnado estrategias y herramientas para la evitación del plagio académico. Dentro de estas, evidentemente, se incluirían aspectos relativos a la promoción de competencias éticas en el manejo y uso de la información; competencias en las que quede reflejada claramente la necesidad de citar y referenciar las fuentes utilizadas, ya sean estas electrónicas o en formato impreso.

- Ofrecer a los docentes los instrumentos necesarios no solo para la correcta identificación de casos de deshonestidad académica y las medidas de corrección de estos (aspecto este muy relacionado con la existencia de una normativa clara relativa a la comisión de fraude académico y la ejecución de medidas en contra de este en los centros educativos), sino también y principalmente para la prevención de este tipo de acciones: metodologías de evaluación alternativas a las ya implementadas, modelos de buenas prácticas para la evitación del plagio académico, herramientas didácticas para la potenciación de la alfabetización informacional entre su alumnado desde los primeros cursos de Educación Secundaria, así como elementos útiles para alcanzar óptimos niveles de coordinación docente, factor este que consideramos se debe potenciar para evidenciar ante el alumnado una coherencia tanto en el planteamiento como en la evaluación de los trabajos académicos.

\section{REFERENCIAS BIBLIOGRÁFICAS}

Branch, A. (2001). SURVEY: As pressures increase, more high school students turn to cheating. Curriculum Administrator, vol. 37 (8). Retrieved April 10, 2014 from http://connection.ebscohost. com/c/articles/4970052/survey-as-pressures-increase-more-high-school-students-turn-cheating

Comas, R., Sureda, J., Angulo, F. \& Mut, T. (2011). Academic plagiarism amongst secondary education students: State of the art. ICERI2011 Proceedings $4^{\text {th }}$ International Conference of Education, Research and Innovations (pp. 4314-4321). Madrid: International Association of Technology, Education and Development.

., Sureda, J., Casero, A. y Morey, M. (2011). La integridad académica entre el alumnado universitario español. Estudios Pedagógicos, vol. 37 (1), 207-225. Recuperado el 10 de abril de 2014 desde http://www.scielo.cl/scielo.php?pid=S0718-07052011000100011\&script=sci_arttext

(2010). Academic plagiarism: Explanatory factors from students' perspective. Journal of Academic Ethics, vol. 8 (3), 217-232. Retrieved April 10, 2014 from http://link.springer.com/ar ticle/10.1007\%2Fs10805-010-9121-0

\& Salvà, F. (2010). Academic plagiarism prevalence among Spanish undergraduate students: An exploratory analysis. Biochemia Medica, vol. 20 (3), 301-306. Retrieved April 10, 2014 from http://www.biochemia-medica.com/content/academic-plagiarism -prevalence-among-spanish-undergraduate-students-exploratory-analysis

(2009). El ciberplagio y otras formas de deshonestidad académica entre el alumnado universitario. Tesis doctoral. Islas Baleares: Universidad de las Islas Baleares.

. y Sureda, J. (2007). Ciber-plagio académico. Una aproximación al estado de los conocimientos. Revista Textos de la Ciber Sociedad, (10). Recuperado el 10 de abril de 2014 desde http://www.cibersociedad.net/textos/articulo.php?art=121 
Coren, A. (2011). Turning a blind eye: Faculty who ignore student cheating. Journal of Academic Ethics, vol. 9 (4), 291-305. Retrieved April 10, 2014 from http://link.springer.com/ article/10.1007\%2Fs10805-011-9147-y

Davis, S. F., Drinan, P. F. \& Bertram-Gallant, T. (2009). Cheating in school: What we know and what we can do. Malden, MA: Wiley-Blackwell.

DeBell, M. \& Chapman, C. (2003). Computer and internet use by children and adolescents in the United States, 2001 (NCES 2004-014). Washington, DC: U.S. Department of Education, National Center for Educations Statics.

Embleton, K. \& Helfer, D. S. (2007). The plague of plagiarism and academic dishonesty. Searcher, vol. 15 (6), 23-26. Retrieved April 10, 2014 from http://scholarworks.csun.edu/bitstream/ handle/10211.2/1674/EmbltonHelfer200707.pdf?sequence=3

Ercegovac, Z. \& Richardson, J. V. (2004). Academic dishonesty, plagiarism included, in the digital age: A literature review. College and Research Libraries, vol. 65 (4), 301-318. Retrieved April 10, 2014 from https://www.baruch.cuny.edu/facultyhandbook/documents/PlagiarismLiteratureReview.pdf

Hexham, I. (1999). Academic plagiarism defined. Retrieved April 10, 2014 from http://people. ucalgary.ca/ hexham/content/articles/plague-of-plagiarism.html

Jordan, A. (2001). College student cheating: The role of motivation, perceived norms, attitudes, and knowledge of institutional policy. Ethics \& Behavior, vol. 11 (3), 233-247. Retrieved April 10, 2014 from http://citeseerx.ist.psu.edu/viewdoc/download?doi=10.1.1.458.4672\&rep=rep1\&type=pdf

Kisamore, J. L., Stone, T. H. \& Jawahar, I. M. (2007). Academic integrity: The relationship between individual and situational factors on misconduct contemplations. Journal of Business Ethics, vol. 75 (4), 381-394. Retrieved April 10, 2014 from http://link.springer.com/article/10.1007/ s10551-006-9260-9

Lambert, E. G., Hogan, N. L. y Barton, S. M. (2003). Collegiate academic dishonesty revisited: What have they done, how often have they done it, who does it, and why did they do it? Electronic Journal of Sociology, vol. 7 (4). Retrieved April 10, 2014 from http://www.sociology.org/content/ vol7.4/ lambert_etal.html

Ma, H. J, Wan, G. \& Lu, E. Y. (2008). Digital cheating and plagiarism in the schools. Theory into Practice, vol. 47 (3), 197-203. Retrieved April 10, 2014 from http://ww2.coastal.edu/jwinslow/ tech/files/readings/digitalcheating.pdf

Ma, Y., McCabe, D. L. \& Liu, R. (2013). Student's academic cheating in Chinese universities: Prevalence, influencing factors, and proposed action. Journal of Academic Ethics, vol. 11 (3), 169184. Retrieved April 10, 2014 from http://link.springer.com/article/10.1007\%2Fs10805-013-9186-7

McCabe, D. L. \& Katz, D. (2009). Curbing cheating. Education Digest, vol. 75 (1), 16-19. Retrieved April 10, 2014 from http://eric.ed.gov/?id=EJ857701

., Trevino, L. \& Butterfield, K. (2001). Cheating in academic institutions: A decade of research. Ethics \& Behavior, vol. 11 (3), 219-232. Retrieved April 10, 2014 from http://www. middlebury.edu/media/view/257513/original/Decade_of_Research.pdf

. (1993). Academic dishonesty: Honor codes and other contextual influences. Journal of Higher Education, vol. 64 (5), 522-538. Retrieved April 10, 2014 from http://library.uvm. edu/ pmardeus/honors/honorcode.pdf

Morey, M., Sureda, J., Oliver, M. y Comas, R. (2013). Plagio y rendimiento académico entre el alumnado de educación secundaria obligatoria. Estudios sobre Educación, (24), 225-244. Recuperado el 10 de abril de 2014 desde http://dadun.unav.edu/handle/10171/29571

Mut, B. (2012). La alfabetización informacional: Una aproximación al ciber-plagio académico entre el alumnado de bachillerato. Tesis doctoral. Islas Baleares: Universidad de las Islas Baleares.

Park, C. (2003). In other (people's) words: Plagiarism by university students-literature and lessons. Assessment \& Evaluation in Higher Education, vol. 28 (5), 471-488. Retrieved April 10, 2014 from http://dutmoodle.dut.ac.za/moodle/pluginfile.php/30664/mod_resource/content/0/ In_other_people_s_words_-_plagiarism_by_university_students_-_literature_and_lessons.pdf 
Pupovac, V., Bilic-Zulle, L. \& Petrovecki, M. (2008). On academic plagiarism in Europe. An analytic approach based on four studies. Digithum, (10). Retrieved April 10, 2014 from http://www. uoc.edu/digithum/10/dt/eng/pupovac_bilic-zulle_petrovecki.pdf

Robelen, E. W. (2007). Online anti-plagiarism service sets off court fight. Education Week, vol. 26 (36). Retrieved April 10, 2014 from http://www.edweek.org/ew/articles/2007/05/09/36pla giarism.h26.html

Sureda, J., Comas, R. y Gili, M. (2009). Prácticas académicas deshonestas en el desarrollo de exámenes entre el alumnado universitario español. Estudios sobre Educación, (17), 103-122. Recuperado el 10 de abril de 2014 desde http://dadun.unav.edu/handle/10171/9852

. y Morey, M. (2009). Las causas del plagio académico entre el alumnado universitario español según el profesorado. Revista Iberoamericana de Educación, (50), 197-220. Recuperado el 10 de abril de 2014 desde http://www.rieoei.org/rie50a10.pdf

Villano, M. (2006). Fighting plagiarism: Taking the work out of homework. The Journal, vol. 33 (15), 24-30. Retrieved April 10, 2014 from http://www.editlib.org/p/77075/

Volpe, R., Davidson, L. \& Bell, M. C. (2008). Faculty attitudes and behaviors concerning student cheating. College Student Journal, vol. 42 (1), 164-175. Retrieved April 10, 2014 from http://eric.ed.gov/?id=EJ816876

Zopatis, A. A. \& Krambia-Kapardis, M. (2008). Ethical behavior of tertiary education students in Cyprus. Journal of Business Ethics, vol. 81 (3), 647-663. Retrieved April 10, 2014 from http:// link.springer.com/article/10.1007\%2Fs10551-007-9538-6 